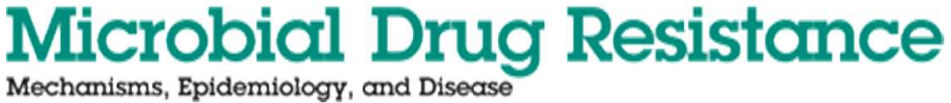

Microbial Drug Resistance: http://mc.manuscriptcentral.com/mdr

\section{Relationship between biofilm formation and antimicrobial resistance in Gram-negative bacteria}

\begin{tabular}{|c|c|}
\hline Journal: & Microbial Drug Resistance \\
\hline Manuscript ID & MDR-2018-0027.R1 \\
\hline Manuscript Type: & Epidemiology \\
\hline Date Submitted by the Author: & $\mathrm{n} / \mathrm{a}$ \\
\hline Complete List of Authors: & $\begin{array}{l}\text { Cepas, Virginio; Instituto de Salud Global Barcelona } \\
\text { López, Yuly; Barcelona Institute for Global Health (ISGlobal) - Hospital } \\
\text { Clínic-Universitat de Barcelona, ; ISGLOBAL } \\
\text { Muñoz, Estela; Instituto de Salud Global Barcelona } \\
\text { Rolo, Dora; Instituto de Salud Global Barcelona } \\
\text { Ardanuy, Carmen; Hospital Universitari de Bellvitge, Microbiology; CIBER } \\
\text { de Enfermedades Respiratorias, } \\
\text { Marti, Sara; Hospital Universitari Bellvitge, Microbiology } \\
\text { Xercavins, Mariona; CatLab, Hospital Mutua de Terrasa } \\
\text { Horcajada, Juan Pablo; Hospital del Mar } \\
\text { Bosch, Jordi; University of Barcelona, } \\
\text { Soto, Sara; Barcelona Institute for Global Health (ISGlobal) - Hospital } \\
\text { Clínic-Universitat de Barcelona, }\end{array}$ \\
\hline Keyword: & $\begin{array}{l}\text { E. Coli, Klebsiella pneumoniae, Minimum Inhibitory Concentrations (MICs), } \\
\text { Pseudomonas aeruginosa, Resistance }\end{array}$ \\
\hline $\begin{array}{r}\text { Manuscript Keywords (Search } \\
\text { Terms): }\end{array}$ & Biofilm, Gram-negative, infections \\
\hline Abstract: & $\begin{array}{l}\text { Gram-negative microorganisms are a significant cause of infection in both } \\
\text { community and nosocomial settings. The increase, emergence and spread } \\
\text { of antimicrobial resistance among bacteria is one of the most important } \\
\text { health problems worldwide. One of the mechanisms of resistance used by } \\
\text { bacteria is biofilm formation which is also a mechanism of virulence. This } \\
\text { study analyzed the possible relationship between antimicrobial resistance } \\
\text { and biofilm formation among isolates of three Gram-negative bacteria } \\
\text { species. Several relationships were found between the ability to form } \\
\text { biofilm and antimicrobial resistance, being different for each species. } \\
\text { Indeed, gentamicin and ceftazidime resistance was related to biofilm } \\
\text { formation in Escherichia coli, piperacillin/tazobactam and colistin in } \\
\text { Klebsiella pneumoniae, and ciprofloxacin in Pseudomonas aeruginosa. } \\
\text { However, no relationship was observed between global resistance or } \\
\text { multidrug-resistance and biofilm formation. In addition, compared to other } \\
\text { reported data, the isolates in the present study showed higher rates of } \\
\text { antimicrobial resistance. In conclusion, the acquisition of specific } \\
\text { antimicrobial resistance can compromise or enhance biofilm formation in } \\
\text { several species of Gram-negative bacteria. However, multidrug-resistant } \\
\text { isolates do not show a trend to being greater biofilm producers than non- }\end{array}$ \\
\hline
\end{tabular}




\section{SCHOLARONE ${ }^{\text {'N }}$} Manuscripts multiresistant isolates.

(1)

(4)

(1)

8

(19)

22

(23

(7)

(8)

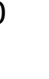

3
9 0

42
45 9 (a) (a)

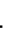
6 7 8 60 
1 Relationship between biofilm formation and antimicrobial resistance in Gram-

2 negative bacteria

3 Virginio Cepas ${ }^{2}$, Yuly López ${ }^{2}$, Estela Muñoz ${ }^{2}$, Dora Rolo ${ }^{2}$, Carmen Ardanuy ${ }^{3}$, Sara

4 Martí $^{3}$, Mariona Xercavins ${ }^{4}$, Juan Pablo Horcajada ${ }^{5}$, Jordi Bosch ${ }^{1,2}$, Sara M. Soto ${ }^{1,2}$

$5{ }^{1}$ Department of Microbiology, Hospital Clínic - Universitat de Barcelona, Barcelona, 6 Spain. ${ }^{2}$ ISGlobal, Barcelona Ctr. Int. Health Res. (CRESIB), Hospital Clínic 7 Universitat de Barcelona, Barcelona, Spain. ${ }^{3}$ Department of Microbiology, Hospital 8 Universitari de Bellvitge, IDIBELL, Universitat de Barcelona, Barcelona, Spain; 9 CIBERes (CIBER de Enfermedades Respiratorias), ISCIII, Madrid, Spain. ${ }^{4}$ Hospital 10 Mutua de Terrassa, Terrassa, Spain. ${ }^{5}$ Hospital del Mar, Barcelona, Spain.

$13{ }^{\#}$ Corresponding author:

14 Sara M. Soto

15 ISGlobal

16 Edificio CEK-1 ${ }^{\text {a }}$ planta; C/ Roselló 149-153

17 08036-Barcelona, Spain

18 Phone: +34-932275707; Fax: +34-932279327

19 e-mail: sara.soto@isglobal.org 


\section{INTRODUCTION}

22 The rise in the emergence and spread of antimicrobial resistance among the different 23 microorganisms (bacteria, fungi, virus, and parasites) is one of the most important 24 health problems worldwide today. Resistance to antibiotics is increasing at both 25 community and hospital levels, being especially relevant in hospital settings in which 26 strong selective pressure favors the selection, persistence and maintenance of resistant, 27 multi-drug-resistant (MDR) and even pan-resistant strains (resistant to all the current 28 groups of antibiotics for therapeutic use) causing antibiotic treatment failure, increased 29 mortality and morbidity, and having a significant impact on the cost of medical 30 treatment and prevention of bacterial infectious diseases. ${ }^{1,2}$ It has been estimated that 31 the annual cost due to antimicrobial-resistant Staphylococcus aureus infections is about $32 \$ 4.6$ billion only in USA. ${ }^{3}$

33 Bacterial resistance to antibiotics is primarily the consequence of a variety of 34 phenomena such as alteration of the target of the drug, impermeability of the bacteria to 35 the antibiotic, and genetically-associated changes (mutational events, genetic transfer of 36 resistance genes via plasmids, and mutations of target genes). ${ }^{4}$ However, this is not the 37 only reason for antimicrobial treatment failure. In fact, the ability to form communities 38 called biofilms embedded in an exopolysaccharide matrix is one of the mechanisms of 39 resistance used by bacteria to survive in the presence of an antibiotic. ${ }^{5}$ In this state, 40 bacteria can be up to 1,000 -fold more resistant to antibiotics than those in a planktonic 41 state. $^{6-8}$ Several studies recommend combined antibiotic therapy as the treatment of 42 choice in biofilm-associated infections caused by Gram-negative bacteria, with 43 macrolides (erythromycin, clarithromycin and azithromycin) being the main antibiotics 44 chosen due to their high antibiofilm activity in vitro and in vivo. ${ }^{9}$ However, antibiotic 45 treatment of biofilm-associated infections requires further study, since the selection of a 
46 specific treatment is difficult because of the wide variability of the microorganisms

47 involved.

48 Several studies have demonstrated that low doses of certain antibiotics can induce

49 biofilm formation indicating that biofilm regulation includes the presence of antibiotics.

50 However, the correlation between biofilm formation and antibiotic resistance is

51 currently unclear and remains under investigation. ${ }^{10,11}$

52 Previous studies carried out in our laboratory showed a relationship between the 53 acquisition of resistance (specifically resistance to quinolones) and the ability to form 54 biofilm $^{12}$ among uropathogenic Escherichia coli (UPEC). It was found that a decrease 55 in biofilm formation was mainly due to a decrease of type 1 fimbriae expression ${ }^{13}$. 56 However, more studies are needed to elucidate this relationship in other bacteria.

57 Thus, the aim of this study was to analyze the possible relationship between the ability 58 to form biofilm and antimicrobial resistance among susceptible, resistant and multidrug59 resistant Gram-negative clinical isolates from different hospitals in Catalonia. 


\section{MATERIAL AND METHODS}

62 Bacteria. Four hundred eight bacterial isolates were collected from four Catalan 63 hospitals (Hospital Clinic of Barcelona, Hospital Universitario de Bellvitge, Hospital 64 del Mar, and Hospital Universitario Mutua de Terrassa) over a 6-month period from 65 2016-2017. Among these, 142 were E. coli, 117 Klebsiella pneumoniae, and 149 were 66 Pseudomonas aeruginosa. The bacteria were isolated from blood, urine and respiratory 67 (including, sputum and tracheal aspirate) samples and processed at the corresponding 68 Microbiology Laboratory. All the isolates were confirmed by matrix-assisted laser 69 desorption ionization-time of flight mass spectrometry (MALDI-TOF) and were stored 70 in skim milk (BD) at $-80^{\circ} \mathrm{C}$. The samples used in our study were sourced through 71 institutional tissue repositories.

72 Analysis of antimicrobial resistance. Resistance profiles were determined using the 73 standard Kirby-Bauer disk-diffusion method following the Clinical \& Laboratory 74 Standards Institute (CSLI) guidelines. ${ }^{14}$ E. coli ATCC 25922 and P. aeruginosa ATCC 7527853 strains were used as controls. The antimicrobial agents tested were: amikacin (30 $76 \mu \mathrm{g})$, amoxicillin/clavulanic acid $(30 \mu \mathrm{g})$, ceftazidime $(30 \mu \mathrm{g})$, cefepime $(30 \mu \mathrm{g})$, 77 imipenem $(10 \mu \mathrm{g})$, meropenem $(10 \mu \mathrm{g})$, trimethoprim-sulfamethoxazole $(30 \mu \mathrm{g})$, 78 gentamicin $(10 \mu \mathrm{g})$, tobramycin $(10 \mu \mathrm{g})$, ciprofloxacin $(5 \mu \mathrm{g})$, chloramphenicol $(30 \mu \mathrm{g})$, 79 aztreonam $(15 \mu \mathrm{g})$, piperacillin/tazobactam $(100 / 10 \mu \mathrm{g})$, fosfomycin $(200 \mu \mathrm{g})$, 80 tigecycline $(15 \mu \mathrm{g})$ and colistin $(10 \mu \mathrm{g})$.

81 Biofilm formation. Biofilm formation was analyzed using a modified protocol 82 previously described by O'Toole et $\mathrm{al}^{15}$. Briefly, all isolates were cultured in aerobic 83 conditions in Luria Bertani (LB) agar (Condalab) for $24 \mathrm{~h}$ at $37^{\circ} \mathrm{C}$ to obtain single 
84 colonies. These colonies were established by the direct colony suspension method in LB 85 broth for $24 \mathrm{~h}$ at $37^{\circ} \mathrm{C}$ with shaking at $180 \mathrm{rpm}$.

86 The Biofilm formation assay was tested in 96-well microtiter plates using an 87 appropriate medium, M63 medium in E. coli strains and LB for P. aeruginosa and $K$. 88 pneumoniae, both mediums supplemented with $0.25 \%$ glucose. The plates were 89 inoculated with the overnight culture diluted 1:100 in fresh medium and incubated for $9024 \mathrm{~h}$ at $37^{\circ} \mathrm{C}$ or $24 \mathrm{~h}$ at $30^{\circ} \mathrm{C}$ in case of E. coli strains, both in static conditions. The final 91 volume of liquid in each well was $200 \mu \mathrm{L}$. All plates include a sterility control (culture 92 medium without inoculum) and a growth control (control medium with inoculum). To 93 avoid evaporation, all plates were covered with adhesive foil lids.

94 The biofilm formation assay for P. aeruginosa was performed using the Calgary 95 protocol as described previously ${ }^{16}$ ). The bacterial biofilm was formed by immersing the 96 pegs of a modified polystyrene microtiter lid into a 96 -well microtiter plate containing $97200 \mu \mathrm{L}$ of the ON culture diluted 1:100 in fresh LB medium (catalog no. 445497; Nunc 98 TSP system, Nunc, Roskilde, Denmark).

\section{Biofilm quantification}

100 After incubation, liquid culture was carefully removed and washed once with $210 \mu \mathrm{L}$ of 101 PBS and dried at $65^{\circ} \mathrm{C}$ until complete desiccation. Biofilms were stained with $200 \mu \mathrm{L}$ of $1021 \%(\mathrm{v} / \mathrm{v})$ solution of crystal violet (CV) stain and incubated $10 \mathrm{~min}$ at room 103 temperature. Afterwards, CV stain was completely removed, washing once with $210 \mu \mathrm{L}$ 104 of PBS and heat-fixed at $65^{\circ} \mathrm{C}$ for $60 \mathrm{~min}$. 
106 The CV was eluted by the addition of $200 \mu \mathrm{L}$ of $33 \%$ glacial acetic acid. The optical 107 density (OD) was measured at $580 \mathrm{~nm}$ using a Microplate reader (EPOCH 2 microplate 108 reader, BioTek, VT, USA).

\section{Biofilm classification}

110 In this study, the heterogeneity in the biomass of the samples requires definition of a 111 cut-off value that would divide the samples in non-adherent, weakly, moderately and 112 strongly-adherent. For this reason, all samples were tested in triplicate and calculated 113 the OD average using negative controls (medium without inoculum). The cut-off value 114 was defined for each species. For easier interpretation of the results, strains were 115 classified into the following categories using an adaptation of a previous study ${ }^{17}$ ):

116 The isolates were categorised in quartiles according OD value using Graphpad Prism 5. 117 The quartile below $25 \%$ percentile were classed as non-adherent $118(\mathrm{OD} 580=0.0640,0.1605$ and 0.3145 for E. coli, K. pneumoniae and P. aeruginosa, 119 respectively). If their biomass absorbances were compressed between $25 \%$ percentile 120 and Median (0.1920, 0.2560, 0.5560 for E. coli, K. pneumoniae and P. aeruginosa, 121 respectively) as weakly adherent. Value between the median and $75 \%$ Percentile $122(0.4165,0.3765,0.8080$ for E. coli, K. pneumoniae and P. aeruginosa, respectively) 123 were classified as moderate adherent and the isolate with OD over $75 \%$ percentile were 124 deemed as strong biofilm producer. According to OD value of positive control of each 125 microorganism were categorized as strong biofilm.

126 Statistical analysis. Chi-square test and Spearman's rank correlation test was 127 performed by SPSS 24.0 for Windows) were used for study de association and 128 correlation between biofilm formation among and antimicrobial susceptibility 129 categories and the respective origin of microorganisms 
Cepas

130

131

132

133

134

135

136

137

138

139

140

141

142

143

144

145

146

147

148

149

150

151

152

153

154

\section{RESULTS}

Approximately $40 \%$ of all the isolates studied were resistant to ciprofloxacin. In addition, $50 \%$ of the $E$. coli isolates were resistant to cotrimoxazol, $36 \%$ of $K$. pneumoniae were resistant to ceftazidime, and about $30 \%$ of the $P$. aeruginosa isolates were resistant to imipenem, meropenem, azthreonam and fosfomycin (Figure 1). According to the number of antibiotic families to which the isolates were resistant, they were classified into susceptible ( $\mathrm{S}$ - not resistant to any family), resistant ( $\mathrm{R}$-resistant to 1-2 categories), multidrug-resistant (MDR - resistant to 3 or more antibiotic families) and extensively drug-resistant (XDR - non-susceptible to at least one agent in all but two or fewer antimicrobial categories (i.e., bacterial isolates remained susceptible to only one or two categories)) Thus, $35 \%$ of all the isolates were $\mathrm{S}, 35 \%$ were $\mathrm{R}$, and $30 \%$ were MDR (data not shown). Among the $E$. coli isolates, $29 \%$ were S, $41 \% \mathrm{R}$ and $30 \%$ MDR. In the case of $K$. pneumoniae, $29 \%, 33 \%$ and $38 \%$ were $\mathrm{S}, \mathrm{R}$ and MDR, respectively. Finally, $41 \%, 31 \%, 19 \%$ and $9 \%$ of $P$. aeruginosa isolates were $\mathrm{S}, \mathrm{R}$, MDR and XDR (Figure 1).

On analysis of the antimicrobial resistance of each species according to the type of sample (blood, respiratory and urine), several differences were found. K. pneumoniae isolates collected from blood were less resistant to fosfomycin than those collected from sputum and urine (1.7\% vs. $9.4 \%$ and $13.7 \%$, respectively). $P$. aeruginosa isolates collected from respiratory were, in general, more resistant to all the antimicrobial agents studied in common in the three species than their counterparts isolated from blood and urine (Table 1).

We studied the ability of all the isolates collected to form biofilm in vitro and found that $49.3 \%$ were able to do so: $30.3 \%$ of the E. coli, $37.6 \%$ of $K$. pneumoniae and $76.5 \%$ of P. aeruginosa isolates, respectively. 
155 No significant differences were found in the frequency of biofilm forming isolates in 156 relation to each type of sample (blood, sputum and urine). However, some trends were 157 observed. For example, in the case of E. coli, the isolates collected from respiratory 158 were less biofilm forming than those collected from blood or urine On the other hand, 159 the $P$. aeruginosa isolates collected from respiratory were more biofilm forming than 160 those from the other types of samples (Figure 2)

161 Relationships between the ability to form biofilm and antimicrobial resistance were 162 scarce and differed for each species. In the case of $K$. pneumoniae, the isolates resistant 163 to colistin showed a strong capacity to form biofilm than the susceptible isolates $(\mathrm{p}=$ 164 0.026) and the biofilm formation was strong in $P$. aeruginosa isolates susceptible to 165 ciprofloxacin than in their resistant counterparts $(\mathrm{p}=0.041)$ (Table 1).

166 Finally, there was no significant relationship between global resistance or multidrug167 resistance and biofilm formation. However, the $P$. aeruginosa isolates susceptible to all 168 the antibiotics studied or resistant to only 1 antimicrobial category tended to be more 169 biofilm forming than the MDR and XDR (Figure 3).

\section{DISCUSSION}

173 Gram-negative microorganisms are a significant cause of infection in both community 174 and nosocomial settings. ${ }^{18}$ The emergence of microorganisms resistant to multiple 175 antibiotics used in the treatment of infections has become an important health problem 176 worldwide. The present study analyzed three species of microorganisms included 177 among the ESKAPE pathogens: $K$. pneumoniae and $P$. aeruginosa, as well as $E$. coli 178 isolates. 
179 The percentage of isolates resistant to the different antibiotics studied was higher in 180 comparison with other studies (Table 3).

181 It was of note that the hospitals participating in this study showed higher rates of 182 ciprofloxacin resistance ranging from $37 \%$ to $45 \%$ compared to other studies reporting a 183 rate of resistance of less than $29 \%$. The high percentage of resistance found among the 184 isolates collected from blood in the hospitals participating in the study could be due to 185 the fact that patients had received antimicrobial treatment before the sample was 186 obtained. It is also well known that the misuse of antibiotics leads to selective pressure 187 that favors the acquisition of resistance. We evaluated the possible relationship between 188 antimicrobial resistance and the ability to form biofilm among the collected isolates. No 189 relationship was found between multidrug-resistance and biofilm formation, but similar 190 to other studies ${ }^{19}$ we found a comparable level of biofilm production in both multidrug 191 and non-multidrug resistant isolates with no significant differences between the two 192 groups. High rates of biofilm producing $K$. pneumoniae have been reported in 193 multidrug-resistant strains, mainly ESBL producers harboring bla $_{\text {CTX-M genes. }}{ }^{20}$

194 However, there are reports regarding relationships between biofilm formation and 195 resistance to specific antibiotics. Thus, the acquisition of quinolone resistance has been 196 related to a decrease in biofilm production in both uropathogenic E. coli and Salmonella 197 typhimurium. ${ }^{12,21}$ In the present study, we also found this relationship between 198 quinolone resistance and biofilm formation in $P$. aeruginosa, with the susceptible 199 isolates showing a greater capacity to form biofilm than the resistant isolates. However, 200 there are discrepancies among the different studies in the literature. One example of this 201 is the study of the effect of meropenem resistance on biofilm formation. Several studies 202 found that the strains resistant to meropenem showed Gram-negative bacteria to have a 203 greater capacity to form biofilm ${ }^{22}$ in contrast to other studies that found an inverse 
204 relationship between meropenem resistance and biofilm formation among other Gram205 negative bacteria such as Acinetobacter baumannii. ${ }^{23}$ Resistance to imipenem has been 206 associated with less biofilm production in $P$. aeruginosa isolates ${ }^{24}$, although we did not 207 observe this association. This is the first time that a relationship between gentamicin 208 resistance and biofilm formation has been reported in E. coli.

210 In conclusion, the acquisition of specific antimicrobial resistance can compromise or 211 enhance biofilm formation in several species of Gram-negative bacteria. However, 212 multidrug-resistant strains did not tend to have greater biofilm production than non213 multiresistant isolates. Further studies are needed to determine how the acquisition of 214 gentamicin resistance affects biofilm formation. 


\section{ACKNOWLEDGMENTS}

219 This manuscript was funded by the European Union's Horizon 2020 research and 220 innovation program under the NOMORFILM project (grant agreement No 634588) and 221 by the "Fondo de Investigaciones Sanitarias" (PI16/00166) integrated in the "Plan 222 Nacional de I+D+I" and co-funded by the "ISCIII-Subdirección General de 223 Evaluación". ISGlobal is a member of the CERCA Program, Generalitat de Catalunya.

224

\section{AUTHOR DISCLOSURE STATEMENT}

226 No commercial associations that might create a conflict of interest in connection with 227 the submitted manuscript exists. 


\section{REFERENCES}

229 1. Guillemot, D., A.C. Crémieux, and P. Courvalin. 2002. Evolution of antimicrobial

230 resistance: impact on antibiotic use. Semin. Respir. Crit. Care Med. 23:25-28.

231 2. Rice, L.B. 2010. Progress and challenges in implementing the research on ESKAPE 232 pathogens. Infect. Control Hosp. Epidemiol. 31 Suppl 1:S7-10.

233 3. Cassell, G.H., and J. Mekalanos. 2001. Development of antimicrobial agents in the 234 era of new and reemerging infectious diseases and increasing antibiotic resistance. 235 JAMA. 285:601-605.

236 4. Qi, L., H. Li, C. Zhang, B. Liang, J. Li, L. Wang, X. Du, X. Liu, S. Qiu, and H. Song. 237 2016. Relationship between Antibiotic Resistance, Biofilm Formation, and Biofilm238 Specific Resistance in Acinetobacter baumannii. Front. Microbiol. 7:483.

239 5. Donlan, R.M., and J.W. Costerton. 2002. Biofilms: survival mechanisms of clinically 240 relevant microorganisms. Clin. Microbiol. Rev. 15:167-193.

241 6. Mack, D., P. Becker, I. Chatterjee, S. Dobinsky, J.K. Knobloch, G. Peters, H. Rohde, 242 and M. Herrmann. 2004. Mechanisms of biofilm formation in Staphylococcus 243 epidermidis and Staphylococcus aureus: functional molecules, regulatory circuits, and 244 adaptive responses. Int. J. Med. Microbiol. 294:203-212.

245 7. Lewis, K. 2008. Multidrug tolerance of biofilms and persister cells. Curr. Top. 246 Microbiol. Immunol. 322:107-131.

247 8. Lewis, K. 2010. Persister cells. Annu. Rev. Microbiol. 64:357-372.

248 9. Ichimiya, T., K. Takeoka, K. Hiramatsu, K. Hirai, T. Yamasaki, and M. Nasu. 1996. 249 The influence of azithromycin on the biofilm formation of Pseudomonas aeruginosa in 250 vitro. Chemotherapy. 42:186-191. 
251 10. Hoffman, L.R., D.A. D'Argenio, M.J. MacCoss, Z. Zhang, R.A. Jones, and S.I. 252 Miller. 2005. Aminoglycoside antibiotics induce bacterial biofilm formation. Nature. $253 \quad 436: 1171-1175$.

254 11. Kaplan, J.B. 2011. Antibiotic-induced biofilm formation. Int. J. Artif. Organs. $255 \quad 34: 737-751$.

256 12. Soto, S.M., A. Smithson, J.A. Martinez, J.P. Horcajada, J. Mensa, and J. Vila. 2007. 257 Biofilm formation in uropathogenic Escherichia coli strains: relationship with 258 prostatitis, urovirulence factors and antimicrobial resistance. J. Urol. 77:365-368.

259 13. Vila, J., and S.M. Soto. 2012. Salicylate increases the expression of marA and 260 reduces invitro biofilm formation in uropathogenic Escherichia coli by decreasing type 261 1 fimbriae expression. Virulence. 3:280-285.

262 14. Clinical and Laboratory Standards Institute (CLSI). 2014. Performance Standards 263 for Antimicrobial Susceptibility Testing; 24th Informational Supplement, M100-S24.

264 15. O’Toole, G.A., and R. Kolter. 1998. Initiation of biofilm formation in Pseudomonas 265 fluorescens WCS365 proceeds via multiple, convergent signaling pathways: a genetic 266 analysis. Mol. Biol. 28:449-461.

267 16. Ali L, Khambaty F, Diachenko G. 2006. Investigating the suitability of the Calgary 268 biofilm device for assessing the antimicrobial efficacy of new agents. Bioresour 269 Technol. 97(15):1887-1893.

270 17. Sherry, L., Rajendran, R., Lappin, D. F., Borghi, E., Perdoni, F., Falleni, M., ... 271 Ramage, G. (2014). Biofilms formed by Candida albicans bloodstream isolates display 272 phenotypic and transcriptional heterogeneity that are associated with resistance and 273 pathogenicity. BMC Microbiology, 14, 182. http://doi.org/10.1186/1471-2180-14-182 
274 18. Kallen, A.J., and A. Srinivasan. 2010. Current epidemiology of multidrug - resistant 275 gram - negative bacilli in the United States. Infect. Control Hosp. Epidemiol. 31 Suppl $276 \quad 1:$ S51-54.

277 19. Di Domenico, E.G., I. Farulla, G. Prignano, M.T. Gallo, M. Vespaziani, I. Cavallo, 278 I. Sperduti, M. Pontone, V. Bordignon, L. Cilli, A. De Santis, F. Di Salvo, F. Pimpinelli, 279 I. Lesnoni La Parola, L. Toma, and F. Ensoli. 2017. Biofilm is a major virulence 280 determinant in bacterial colonization of chronic skin ulcers independently from the 281 multidrug resistant phenotype. Int. J. Mol. Sci. 18. pii: E1077. 20. Singhai, M., A. Malik, M. Shahid, M.A. Malik, and R. Goyal. 2012. A study on 283 device-related infections with special reference to biofilm production and antibiotic 284 resistance. J. Glob. Infect. Dis. 4:193-198.

285 21. Fàbrega, A., S.M. Soto, C. Ballesté-Delpierre, D. Fernández-Orth, M.t. Jiménez de 286 Anta, and J. Vila. 2014. Impact of quinolone-resistance acquisition on biofilm 287 production and fitness in Salmonella enterica. J. Antimicrob. Chemother. 69:18152881824.

289 22. Mishra, S.K., P. Basukala, O. Basukala, K. Parajuli, B.M. Pokhrel, and B.P. Rijal. 290 2015. Detection of biofilm production and antibiotic resistance pattern in clinical 291 isolates from indwelling medical devices. Curr. Microbiol. 70:128-134.

292 23. Perez, L.R. 2015. Acinetobacter baumannii displays inverse relationship between 293 meropenem resistance and biofilm production. J. Chemother. 27:13-16.

294 24. Musafer, H.K., S.L. Kuchma, A.A. Naimie, J.D. Schwartzman, H.J. Al-Mathkhury, 295 and G.A. O'Toole. 2014. Investigating the link between imipenem resistance and 296 biofilm formation by Pseudomonas aeruginosa. Microb. Ecol. 68:111-120. 


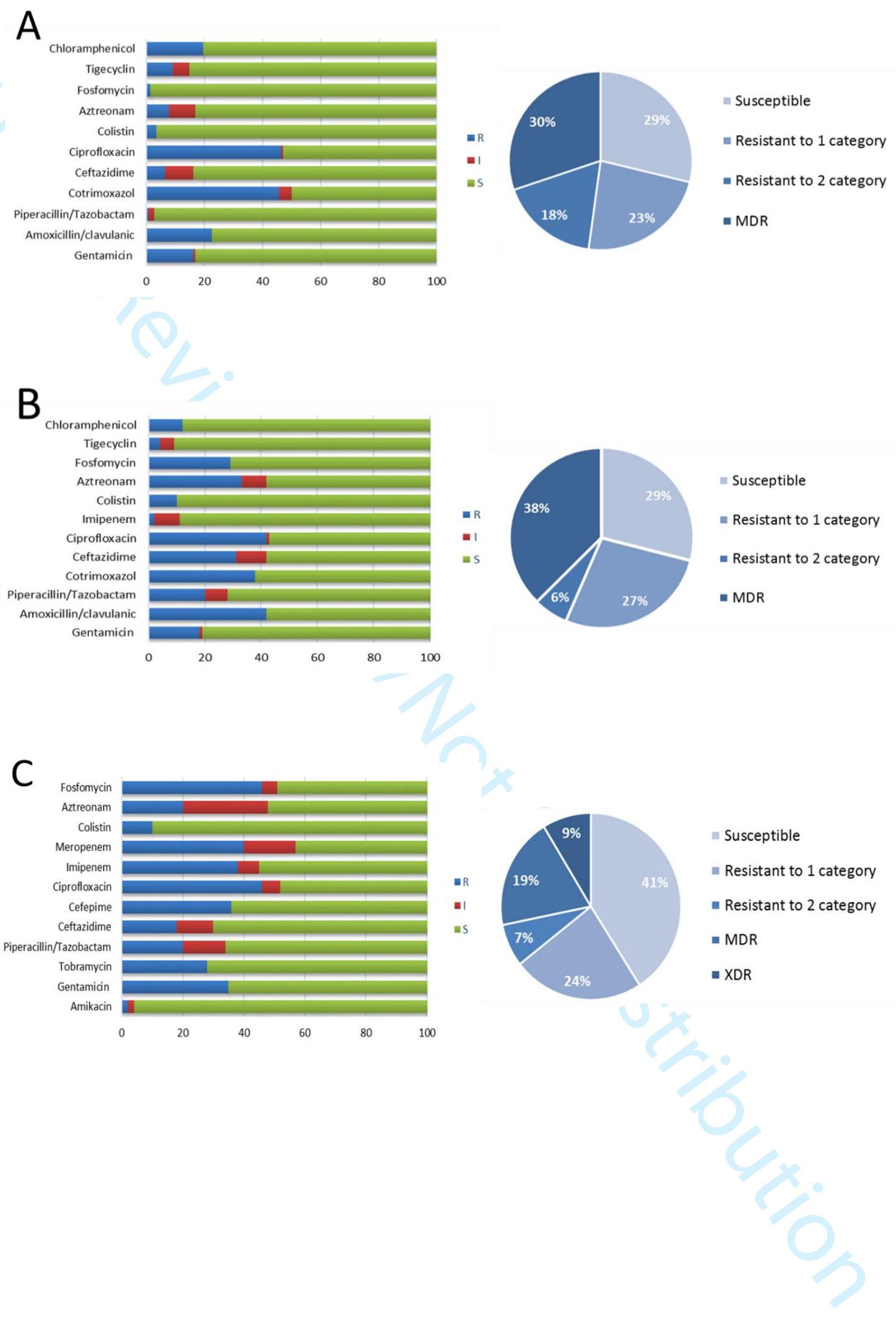


A

STRONGLY-ADHERENT MODERATELY-ADHERENT

WEAKLY-ADHERENT

NON-ADHERENT

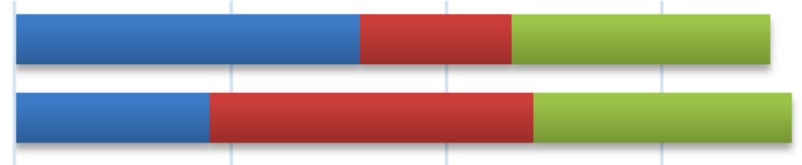

n Bood

- Respiratory

urine
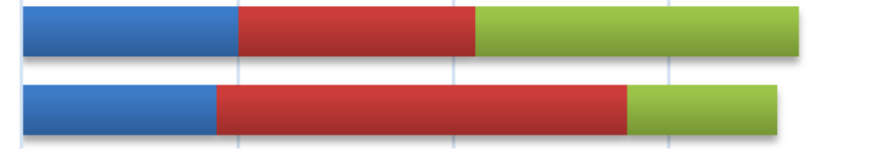

0

10

20

30

40

B

STRONGLY-ADHERENT MODERATELY-ADHERENT

WEAKLY-ADHERENT

NON-ADHERENT

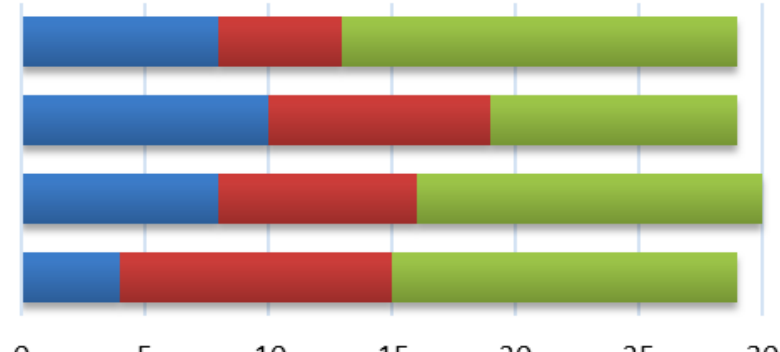

a Bood

- Respiratory

च urine

0

$10 \quad 15 \quad 20$

$25 \quad 30$

35

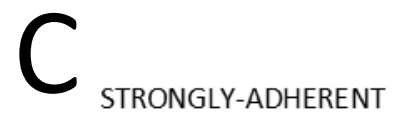

MODERATELY-ADHERENT

WEAKLY-ADHERENT

NON-ADHERENT

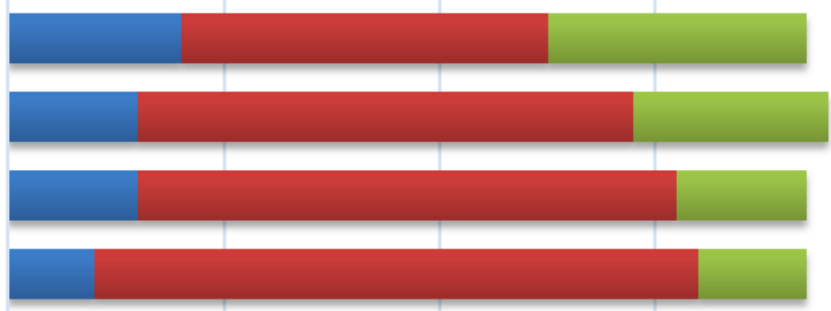

a Bood

- Respiratory

v urine

0

10

20

30

40 

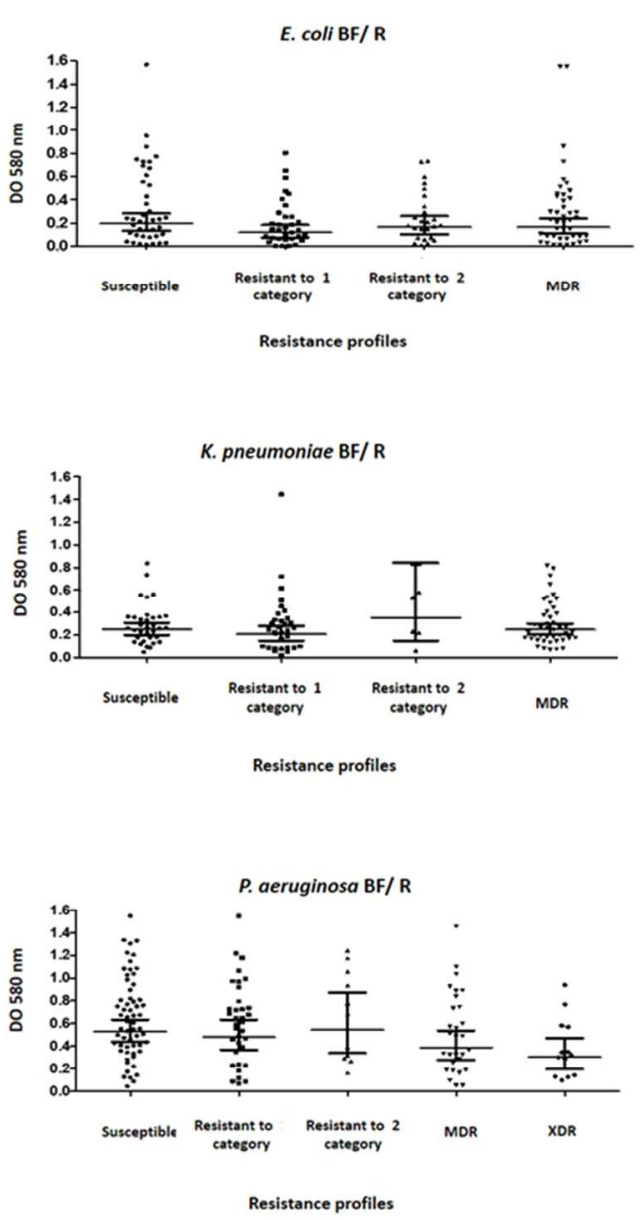

Figure 3

$190 \times 275 \mathrm{~mm}(96 \times 96 \mathrm{DPI})$ 


\section{FIGURE LEGENDS}

2 Figure 1. Percentages of isolates resistant to the different antibiotics used in the

3 treatment of each microorganism (A: E. coli, B: K. pneumoniae, and C: P. aeruginosa)

4 MDR: multidrug-resistant and XDR: extensively drug-resistant.

5

6 Figure 2. Relationship between origin of microorganism and biofilm forming capacities

7

8 Figure 3. Distribution of biofilm formation of isolate with different resistance 9 phenotype.

10 MDR: multidrug-resistant; XDR: extensively drug-resistant.

11 The distribution was separate in quartile according OD580 value. The OD range of 12 positive control biofilm is between $0.8-1$. 
Table 1. Percentage of susceptibly of microorganisms isolate from blood, respiratory and urine against different antimicrobials in common used in this study.

\begin{tabular}{|c|c|c|c|c|c|c|c|c|c|c|c|c|c|}
\hline & & \multicolumn{4}{|c|}{ E. coli } & \multicolumn{4}{|c|}{ K. pneumoniae } & \multicolumn{4}{|c|}{ P. aeruginosa } \\
\hline & & $\mathrm{S} \%$ & $\mathrm{R} \%$ & p-value & $\rho$ & $\mathrm{S} \%$ & $\mathrm{R} \%$ & p-value & $\rho$ & $\mathrm{S} \%$ & $\mathrm{R} \%$ & p-value & $\mathrm{P}$ \\
\hline \multirow[t]{3}{*}{ Ceftazidime } & Blood & 26.8 & 4.2 & & & 16.2 & 9.4 & & & 13.4 & 2.7 & & \\
\hline & Respiratory & 35.2 & 1.4 & & & 20.5 & 7.7 & & & 59.1 & 3.4 & & \\
\hline & Urine & 31.7 & 0.7 & 0.054 & -0.186 & 36.8 & 9.4 & 0.267 & -0.148 & 15.4 & 6 & $0.002 *$ & 0.142 \\
\hline \multirow[t]{3}{*}{ Imipenem } & Blood & 100 & 0 & & & 25.6 & 0 & & & 10.1 & 6 & & \\
\hline & Respiratory & 100 & 0 & & & 16.5 & 1.7 & & & 51 & 11.4 & & \\
\hline & Urine & 100 & 0 & $\mathrm{a}$ & $\mathrm{a}$ & 46.2 & 0 & 0.075 & -0.050 & 13.4 & 8.1 & 0.033* & 0.03 \\
\hline \multirow[t]{3}{*}{ Gentamicin } & Blood & 28.2 & 2.8 & & & 22.2 & 3.4 & & & 10.7 & 5.4 & & \\
\hline & Respiratory & 28.2 & 8.5 & & & 25.6 & 2.6 & & & 51 & 11.4 & & \\
\hline & Urine & 27.5 & 4.9 & 0.175 & 0.063 & 36.8 & 9.4 & 0.344 & 0.103 & 14.8 & 6.7 & 0.152 & 0.007 \\
\hline \multirow[t]{3}{*}{ Ciprofloxacin } & Blood & 16.9 & 14.1 & & 8 & 16.2 & 9.4 & & & 10.7 & 5.4 & & \\
\hline & Respiratory & 16.2 & 20.4 & & & 18.8 & 9.4 & & & 45.6 & 16.8 & & \\
\hline & Urine & 20.4 & 12 & 0.174 & -0.071 & 29.1 & 17.1 & 0.936 & 0.011 & 12.8 & 8.7 & 0.335 & 0.063 \\
\hline \multirow[t]{3}{*}{ Aztreonam } & Blood & 26.8 & 4.2 & & & 17.1 & 8.5 & & & 13.5 & 2.7 & & \\
\hline & Respiratory & 35.5 & 2.1 & & & 20.5 & 7.7 & & & 55.4 & 6.8 & & \\
\hline & Urine & 31 & 1.4 & 0.206 & -0.137 & 34.2 & 12 & 0.762 & -0.062 & 17.6 & 4.1 & 0.471 & 0.032 \\
\hline \multirow[t]{3}{*}{ Piperacillin/tazobactam } & Blood & 31 & 0 & & & 20.5 & 5.1 & 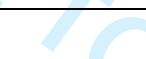 & & 14.1 & 2 & & \\
\hline & Respiratory & 35.9 & 0.7 & & & 18.8 & 11 & & & 55 & 7.4 & & \\
\hline & Urine & 32.4 & 0 & 0.418 & -0.002 & 43.6 & 2.6 & 0.003* & -0.216 & 17.4 & 4 & 0.606 & 0.063 \\
\hline \multirow[t]{3}{*}{ Fosfomycin } & Blood & 31 & 0 & & & 23.9 & 1.7 & & & 8.1 & 8.1 & & \\
\hline & Respiratory & 36.6 & 0 & & & 18.8 & 9.4 & & & 49 & 13.4 & & \\
\hline & Urine & 31 & 1.4 & 0.120 & 0.148 & 32.5 & 13.7 & $\mathbf{0 . 0 2 7} *$ & 0.180 & 12.1 & 9.4 & $0.005 *$ & -0.004 \\
\hline \multirow[t]{3}{*}{ Colistin } & Blood & 28.9 & 2.1 & & & 23.1 & 2.6 & & & 14.8 & 1.3 & & \\
\hline & Respiratory & 35.9 & 0.7 & & & 27.4 & 0.9 & & & 59.7 & 2.7 & & \\
\hline & Urine & 31.7 & 0.7 & 0.360 & -0.099 & 41 & 5.1 & 0.403 & 0.045 & 18.8 & 2.7 & 0.262 & 0.067 \\
\hline
\end{tabular}


Susceptible (S), Resistant (R), Spearman’s rank correlation coefficient ( $\rho$ ), No statistics have been calculated (a).

* Statically significant $(\mathrm{p}<0.05)$. 
Table 2. Relationship between biofilm formation and antimicrobial resistance.

\begin{tabular}{lccc}
\hline \multirow{1}{*}{ Antimicrobials } & \multicolumn{3}{c}{ p-value $(>0.05)$} \\
\cline { 2 - 4 } Amikacin & E. coli & K. pneumoniae & P. aeruginosa \\
Gentamicin & $\mathrm{ND}$ & $\mathrm{ND}$ & 0.561 \\
Tobramycin & 0.133 & 0.826 & 0.254 \\
Amoxicillin/clavulanic & $\mathrm{ND}$ & $\mathrm{ND}$ & 0.607 \\
Piperacillin/Tazobactam & 0351 & 0.713 & $\mathrm{ND}$ \\
Ceftazidime & 0.397 & 0.118 & 0.128 \\
Cefepime & 0.109 & 0.396 & 0.580 \\
Imipenem & $\mathrm{ND}$ & $\mathrm{ND}$ & 0.161 \\
Meropenem & 1 & 0.572 & 0.861 \\
Ciprofloxacin & $\mathrm{ND}$ & $\mathrm{ND}$ & 0.775 \\
Fosfomycin & 0.06 & 0.898 & $\mathbf{0 . 0 4 1 *}$ \\
Aztreonam & 0.113 & 0.148 & 0.935 \\
Colistin & 0.780 & 0.310 & 0.428 \\
Chloramphenicol & 0.639 & $\mathbf{0 . 0 2 6 *}$ & 0.128 \\
Tigecyclin & 0.448 & 0.3 & $\mathrm{ND}$ \\
Cotrimoxazol & 0.669 & 0.098 & $\mathrm{ND}$ \\
\hline ND, Not & 0.783 & 0.667 & \\
\hline & & & \\
\hline
\end{tabular}

ND, Not determined

* Statically significant $(\mathrm{p}<0.05)$. 
Table 3. Percentage of resistance in blood isolates reported in different studies.

\begin{tabular}{|c|c|c|c|c|c|}
\hline \multicolumn{6}{|c|}{ E. coli } \\
\hline & $\begin{array}{c}\text { Yang Q } \\
(2017) \\
{[17]}\end{array}$ & $\begin{array}{c}\text { Guy R } \\
\text { (2016) } \\
{[18]}\end{array}$ & $\begin{array}{c}\text { Bell JM } \\
\text { (2016) } \\
{[19]}\end{array}$ & $\begin{array}{c}\text { Wong PH } \\
(2014) \\
{[20]}\end{array}$ & Present study \\
\hline Gentamicin & - & 9.6 & 7.5 & - & 34.8 \\
\hline Amikacin & 7.12 & - & - & - & - \\
\hline Piperacillin/Tazobactam & 7.49 & 11 & 3.2 & 3.9 & 0.2 \\
\hline Cotrimoxazol & - & - & 29.2 & 34.30 & 15.9 \\
\hline Ceftazidime & - & 11.1 & 4.4 & 24 & 2.2 \\
\hline Ciprofloxacin & - & 18.7 & 10.4 & 28.8 & 16.2 \\
\hline Imipenem & 1.28 & 0.1 & 0.1 & - & 0 \\
\hline \multicolumn{6}{|c|}{ K. pneumoniae } \\
\hline Gentamicin & - & 7.5 & 5.5 & - & 4.4 \\
\hline Amikacin & 12.1 & & - & - & - \\
\hline Piperacillin/Tazobactam & 24.2 & 16.9 & 4.8 & 8.30 & 4.9 \\
\hline Cotrimoxazol & - & - & 15.5 & 12.5 & 9.3 \\
\hline Ceftazidime & - & 12.1 & 6.1 & 13.3 & 7.6 \\
\hline Ciprofloxacin & - & 10.9 & 5 & 16.7 & 10.3 \\
\hline Imipenem & 7.26 & 1.5 & 1.1 & - & 0.5 \\
\hline \multicolumn{6}{|c|}{ P. aeruginosa } \\
\hline Gentamicin & - & - & - & - & 8.6 \\
\hline Amikacin & 25.25 & - & - & - & 0.5 \\
\hline Piperacillin/Tazobactam & 25.59 & - & - & 8 & 4.9 \\
\hline Cotrimoxazol & - & - & - & - & - \\
\hline Ceftazidime & - & 7.4 & - & 12.70 & 4.4 \\
\hline Ciprofloxacin & - & - & - & 21.10 & 11.3 \\
\hline Imipenem & 15.82 & 11.5 & - & 4.20 & 9.3 \\
\hline
\end{tabular}


